\title{
Article
}

\section{The smallest sum-connectivity index on trees with $n$ vertices and $k$ pendant vertices}

Yuedan $\mathrm{Yao}^{1, *}$

1 Department of Mathematics, South China Agricultural University, Guangzhou, 510642, P.R. China

* Correspondence: yaoyuedan12@163.com; Tel.: +8615767973217

Received: 15 April 2019; Accepted: 21 May 2019; Published: 25 June 2019.

\begin{abstract}
For a given connected graph $G$ and a real number $\alpha$, denote by $d(u)$ the degree of vertex $u$ of $G$, and denote by $\chi_{\alpha}(G)=\sum_{u v \in E(G)}(d(u)+d(v))^{\alpha}$ the general sum-connectivity index of $G$. In the present note, we determine the smallest general sum-connectivity index of trees (resp., chemical trees) together with corresponding extremal trees among all trees (resp., chemical trees) with $n$ vertices and $k$ pendant vertices for $0<\alpha<1$.
\end{abstract}

Keywords: General sum-connectivity index, chemical trees, extremal trees.

MSC: 05C05, 05C12, 05C50.

\section{Introduction}

$\mathbf{L}$ et $G=(V, E)$ be a connected undirected simple graph. If $G$ contains exactly $n$ vertices and $n-1$ edges, then $G$ is called a tree. For a vertex $u$ of $G$, denote by $N(u)$ and $d(u)$ the neighborhood set and degree of $u$, that is, $d(u)=|N(u)|$. If $d(u)=k$, then we call $u$ as a $k$-vertex. Specially, a 1-vertex is called a pendant vertex, while a $k$-vertex with $k \geq 2$ will be referred as a non-pendant vertex. A pendant edge is an edge incident with a pendant vertex. Hereafter, $n_{k}$ defines the number of $k$-vertices. Denote by $\Delta(G)$ the maximum degree of $G$. As usual, $S_{n}$ and $P_{n}$ define the star and path with $n$ vertices, respectively.

Let $G-u v$ be the resultant graph obtained after removing the edge $u v$ from the edge set of $G$. Let $E_{2}(G)=$ $\{u v: u v \in E(G)$ with $d(u)=d(v)=2\}$. We write $A:=B$ to indicate that $B$ is the same as $A$.

The first Zagreb index $M_{1}(G)$ and second Zagreb index $M_{2}(G)$ [1] are two famous topological indices among all the vertex-degree-based graph invariants, where

$$
M_{1}(G)=\sum_{v \in V(G)}(d(v))^{2}, \quad \text { and } \quad M_{2}(G)=\sum_{u v \in E(G)} d(u) d(v) .
$$

In what follows, $\alpha$ denotes a real number. As a generalization of $M_{1}(G), \mathrm{Li}$ and Zheng [2] introduced the notation of zeroth-order general Randić index $R_{\alpha}^{0}(G)$, where

$$
R_{\alpha}^{0}(G)=\sum_{v \in V(G)}(d(v))^{\alpha} .
$$

Since $\sum_{u v \in E(G)}(d(u)+d(v))=\sum_{v \in V(G)}(d(v))^{2}$, as another extension to $M_{1}(G)$, the general sum-connectivity index $[3] \chi_{\alpha}(G)$ of $G$ is constructed as

$$
\chi_{\alpha}(G)=\sum_{u v \in E(G)}(d(u)+d(v))^{\alpha} .
$$

Recently, the extremal problems of general sum-connectivity index among different categories of graphs have attracted more and more attention. In this field, Cui et al. [4] obtained the largest general sum-connectivity indices of trees and chemical trees with their corresponding extremal trees among all tree and chemical trees with $n$ vertices and $k$ pendant vertices for $-1 \leq \alpha<0$; Tomescu et al. [5] identified 
the smallest general sum-connectivity index of unicyclic graphs with $n$ vertices and pendant vertices for $-1 \leq \alpha<0$ and charaterized the corresponding extremal graphs; Tomescu et al. [6] determined the smallest general sum-connectivity index of trees with fixed diameter, $n$ vertices and $k$ pendant vertices for $-1 \leq \alpha<0$, respectively.

In what follows, by following the idea of [4], we will demonstrate the smallest general sum-connectivity index with its corresponding extremal trees in the class of trees and chemical trees with $n$ vertices and $k$ pendant vertices for $0<\alpha<1$, respectively.

\section{Preliminaries}

This section is dedicated to some lemmas, which will be used to prove our main result in the next section. For this aim, we need to introduce more notations. It is easily checked that any tree has at least two pendant vertices and the star is the unique tree with $n-1$ pendant vertices and $n$ vertices. For $2 \leq k \leq n-1$, denote by $\mathcal{T}_{n, k}$ (resp., $\mathcal{C} \mathcal{T}_{n, k}$ ) the set of trees (resp., chemical trees) with $n$ vertices and $k$ pendant vertices. If $T \in \mathcal{T}_{n, k}$ and every non-pendant vertex of $T$ is a 3-vertex, then $T$ is called a $(k, 3)$-tree. It can be straightly checked that every $(k, 3)$-tree has $2 k-2$ vertices, among which there is exactly $k$ pendant vertices.

Let $\mathcal{T}_{n, k}^{*}=\{T: T$ is obtained from a $(k, 3)$-tree $H$ by adding $n-2 k+2$ new 2 -vertices to $H$ such that every pendant edge of $H$ is inserted at least one new 2-vertex $\}$. For every tree $T$ of $\mathcal{T}_{n, k^{*}}$, from the definition of $\mathcal{T}_{n, k}^{*}$ we can conclude that $|V(T)|=n, \Delta(T)=3$ and each neighbor vertex of a 3-vertex is either a 3-vertex or a 2-vertex. This implies that $n_{1}(T)=k, n_{2}(T)=n-2 k+2$ and $n_{3}(T)=k-2$. For example, there are exactly trees in the class of $\mathcal{T}_{12,4}^{*}$, which are shown in Figure 1. Combining with $\mathcal{T}_{n, 2}=\mathcal{C} \mathcal{T}_{n, 2}=\left\{P_{n}\right\}, \mathcal{T}_{n, n-1}=\left\{S_{n}\right\}$ and $\mathcal{C} \mathcal{T}_{n, n-1}=\left\{S_{n} \mid 3 \leq n \leq 5\right\}$, we only take $3 \leq k \leq n-2$ into consideration in what follows.
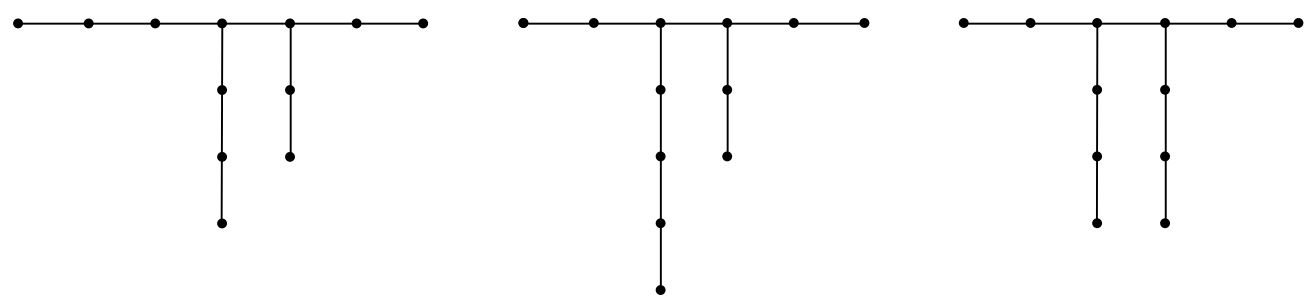

Figure 1. The elements of the set $\mathcal{T}_{12,4}^{*}$.

The following three transformations on a tree $T$ with $n$ vertices were firstly constructed by Zhang et al. [7], they will play an important role in the proof of our main result.

(i) For a fixed edge $u v$ of the tree $T$ with $n$ vertices, denote by $T_{u v}$ the tree obtained from $T$ via identifying the two vertices of $T-u v$, that is, $T_{u v}$ is a tree with $n-1$ vertices obtained from $T$ by contracting the edge $u v$ of $T$. Hereafter, we say that $T_{u v}$ is obtained from $T$ by Transformation I.

(ii) Let $v \in V(T)$ with $N(v)=V^{\prime} \cup V^{\prime \prime}$ satisfying $V^{\prime} \cap V^{\prime \prime}=\varnothing,\left|V^{\prime}\right|=q_{1} \geq 1$ and $\left|V^{\prime \prime}\right|=q_{2} \geq 1$. Denote by $T_{v \mapsto\left(q_{1}, q_{2}\right)}$ the tree with $n+1$ vertices constructed from the tree $T$ via splitting the vertex $v$ into two new vertices $v^{\prime}$ and $v^{\prime \prime}$, adding one edge between $v^{\prime}$ and $v^{\prime \prime}$, joining $v^{\prime}$ and all vertices of $V^{\prime}$, and then joining $v^{\prime \prime}$ and all vertices of $V^{\prime \prime}$. In the sequel, $T_{v \mapsto\left(q_{1}, q_{2}\right)}$ will be referred to be obtained from $T$ by Transformation II.

(iii) Let $v$ be a $q$-vertex of the tree $T$, where $q \geq 4$. Denote by $T_{v \mapsto(3-r e g)}$ the graph with $n+q-3$ vertices obtained from $T$ via replacing the vertex $v$ of $T$ with a $(q, 3)$-tree $H$ such that every vertex of $N(v)$ and every pendant vertex of $H$ are identified individually. In what follows, we say that $T_{v \mapsto(3-r e g)}$ is obtained from $T$ by Transformation III.

Let $T$ be the tree with eight vertices as shown in Figure 2. The readers can refer to Figure 2 for illustrated examples to the above three transformations, where $V^{\prime}=\{u\}$ and $V^{\prime \prime}=\left\{v_{1}, v_{2}, v_{3}\right\}$.

Lemma 1. If $k \geq 1$ and $0<\alpha<1$, then $\varphi(x)=(x+k)^{\alpha}-x^{\alpha}$ is a strictly decreasing function for $x \geq 1$.

Proof. It is easy to see that $\varphi^{\prime}(x)=\alpha\left((x+k)^{\alpha-1}-x^{\alpha-1}\right)<0$ for $k \geq 1$ and $0<\alpha<1$. Consequently, the result holds. 


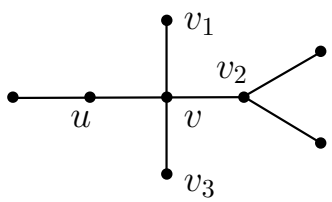

$T$

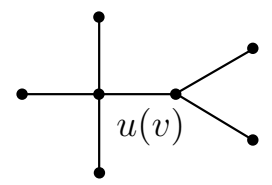

$T_{u v}$

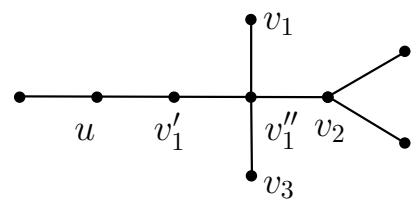

$T_{v \rightarrow(1,3)}$

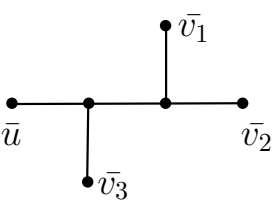

$H$

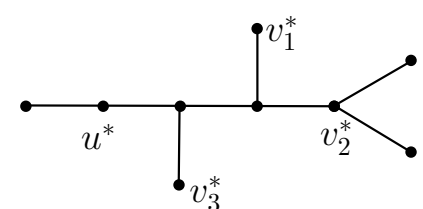

$T_{v \rightarrow(3-r e g)}$

Figure 2. The example of three transformations.

Lemma 2. Let $T$ be a tree of $\mathcal{T}_{n, k}$ and $w$ be a 2-vertex of $T$. If the two neighbor vertices of $w$ are both non-pendant vertices and $0<\alpha<1$, then there is a tree $T^{*}$ of $\mathcal{T}_{n, k}$ such that $\chi_{\alpha}(T) \geq \chi_{\alpha}\left(T^{*}\right)$, with equality iff $w$ is adjacent to at least one 2-vertex.

Proof. Suppose that $N(w)=\{u, v\}$ with $d(u)=s \geq 2$ and $d(v)=t \geq 2$. Let $y$ be a pendant vertex with $x y \in E(G)$ and $d(x)=r \geq 2$. By Transformation I, we can construct $T_{u w}$ from $T$. Let $T^{*}$ be the tree obtained from $T_{u v}$ via adding a new pendant edge adjacent to $x$. Now, it is easily checked that $T^{*}$ is also a tree of $\mathcal{T}_{n, k}$ such that

$$
\begin{aligned}
\chi_{\alpha}(T)-\chi_{\alpha}\left(T^{*}\right) & =(s+2)^{\alpha}+(t+2)^{\alpha}+(r+1)^{\alpha}-(s+t)^{\alpha}-(r+2)^{\alpha}-3^{\alpha} \\
& =\left((s+2)^{\alpha}+(t+2)^{\alpha}-(s+t)^{\alpha}\right)-\left((r+2)^{\alpha}-(r+1)^{\alpha}\right)-3^{\alpha} .
\end{aligned}
$$

Let $\psi(s, t)=(s+2)^{\alpha}+(t+2)^{\alpha}-(s+t)^{\alpha}$ with $s, t \geq 2$. Combining with $0<\alpha<1$, we have

$$
\frac{\partial \psi(s, t)}{\partial s}=\alpha\left((s+2)^{\alpha-1}-(s+t)^{\alpha-1}\right) \geq 0, \text { and } \frac{\partial \psi(s, t)}{\partial t}=\alpha\left((t+2)^{\alpha-1}-(s+t)^{\alpha-1}\right) \geq 0,
$$

this implies that $\psi(s, t)$ is increasing for both $s$ and $t$ with $s, t \geq 2$. In view of Lemma 1 (the case of $k=1$ ), it follows that

$$
\chi_{\alpha}(T)-\chi_{\alpha}\left(T^{*}\right)=\psi(s, t)-\varphi(r+1)-3^{\alpha} \geq \psi(2,2)-\varphi(3)-3^{\alpha}=0 .
$$

So the assertion of the lemma holds.

Lemma 3. Let $T$ be a tree of $\mathcal{T}_{n, k}$ and $x y$ be an edge of $T$ with $d(x)=q \geq 3$ and $d(y)=1$. If $0<\alpha<1$ and $E_{2}(T) \neq \varnothing$, then there is a tree $T^{*}$ of $\mathcal{T}_{n, k}$ with $\chi_{\alpha}(T)>\chi_{\alpha}\left(T^{*}\right)$.

Proof. Note that $E_{2}(T) \neq \varnothing$. Thus, we can construct a new tree $T^{*}$ with $n-1$ vertices from $T$ by contracting an arbitrary edge of $E_{2}(T)$ and then inserting another new vertex to the pendant edge $x y$. Now, $T^{*}$ is also a tree of $\mathcal{T}_{n, k}$. By Lemma 1 (the case of $k=1$ ), it follows that

$$
\begin{aligned}
\chi_{\alpha}(T)-\chi_{\alpha}\left(T^{*}\right) & =4^{\alpha}+(q+1)^{\alpha}-(q+2)^{\alpha}-3^{\alpha} \\
& =\left(4^{\alpha}-3^{\alpha}\right)-\left((q+2)^{\alpha}-(q+1)^{\alpha}\right) \\
& =\varphi(3)-\varphi(q+1)>0 .
\end{aligned}
$$

This finishes the proof of the lemma. 
Lemma 4. Let $T$ be a tree of $\mathcal{T}_{n, k}$ and $u$ be a 4-vertex of $T$. Let $v_{1}, v_{2}, v_{3}$ and $v_{4}$ be four neighbor vertices of $u$ such that $d\left(v_{4}\right) \leq 5$ and $d\left(v_{1}\right) \leq d\left(v_{2}\right) \leq d\left(v_{3}\right) \leq 3$. If $0<\alpha<1$ and $E_{2}(T) \neq \varnothing$, then there is tree $T^{*}$ of $\mathcal{T}_{n, k}$ with $\chi_{\alpha}(T)>\chi_{\alpha}\left(T^{*}\right)$.

Proof. Note that $E_{2}(T) \neq \varnothing$. Thus, we can construct a new tree $T^{\prime}$ with $n-1$ tree from $T$ via contracting an arbitrary edge of $E_{2}(T)$. By Transformation III, we can construct another tree $T^{*}:=T_{v \rightarrow(3-r e g)}^{*}$ from $T^{\prime}$ such that $T^{*} \in \mathcal{T}_{n, k}$. Since $d\left(v_{1}\right) \leq d\left(v_{2}\right) \leq d\left(v_{3}\right) \leq 3, d\left(v_{4}\right) \leq 5$ and in view of Lemma 1 (the case of $k=1$ ), it follows that

$$
\begin{aligned}
\chi_{\alpha}(T)-\chi_{\alpha}\left(T^{*}\right) & =4^{\alpha}+\sum_{i=1}^{4}\left(\left(4+d\left(v_{i}\right)\right)^{\alpha}-\left(3+d\left(v_{i}\right)\right)^{\alpha}\right)-6^{\alpha} \\
& \geq 4^{\alpha}+3\left(7^{\alpha}-6^{\alpha}\right)+\left(9^{\alpha}-8^{\alpha}\right)-6^{\alpha} \\
& =4^{\alpha}-4 \cdot 6^{\alpha}+3 \cdot 7^{\alpha}-8^{\alpha}+9^{\alpha} .
\end{aligned}
$$

Let $\phi(\alpha)=4^{\alpha}-4 \cdot 6^{\alpha}+3 \cdot 7^{\alpha}-8^{\alpha}+9^{\alpha}$ with $0<\alpha<1$. It can be easily checked that $\phi(\alpha)>0$ for $0<\alpha<1$ by applying $M A T L A B$, see Figure 3 (Actually, Figures 3-5 are all drawn by MATLAB).

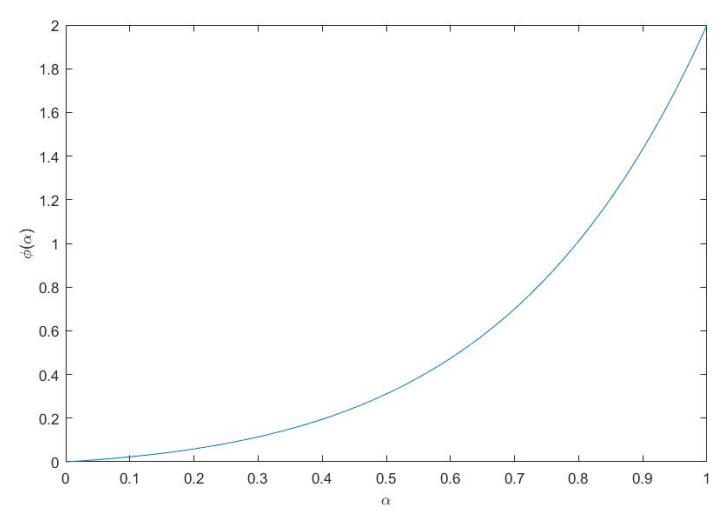

Figure 3. The function $\phi(\alpha)$ in Lemma 4.

Lemma 5. Let $T$ be a tree of $\mathcal{T}_{n, k}$ and $u$ be a q-vertex of $T$, where $q \geq 4$. Suppose that $\left|E_{2}(T)\right| \geq q-3$ and $N(u)=$ $\left\{v_{1}, v_{2}, \ldots, v_{q}\right\}$ with $d\left(v_{1}\right) \leq d\left(v_{2}\right) \leq \cdots \leq d\left(v_{q}\right)$. If $0<\alpha<1$ and $T$ satisfies either $(i)$ or (ii), then there is a tree $T^{*}$ of $\mathcal{T}_{n, k}$ with $\chi_{\alpha}(T)>\chi_{\alpha}\left(T^{*}\right)$, where $(i) d\left(v_{q-1}\right) \leq 3$ and $q \geq 5$; and (ii) $d\left(v_{q-1}\right) \leq 4$ and $q \geq 8$.

Proof. Let $d\left(v_{q-1}\right)=p$. Note that $\left|E_{2}(T)\right| \geq q-3$. Thus, we can construct a new tree $T^{\prime}$ with $n+3-q$ vertices obtained from $T$ by contracting $q-3$ arbitrary edges of $E_{2}(T)$. Taking Transformation III into consideration, we can construct another tree $T^{*}:=T_{v \mapsto(3-r e g)}^{*}$ from $T^{\prime}$. Now, $T^{*}$ is also a tree of $\mathcal{T}_{n, k}$. Since $q \geq 4,0<\alpha<1$ and in view of Lemma 1 (the case of $k=q-3 \geq 1$ ), we have

$$
\begin{aligned}
\chi_{\alpha}(T)-\chi_{\alpha}\left(T^{*}\right)= & (q-3) 4^{\alpha}+\sum_{i=1}^{q}\left(\left(d\left(v_{i}\right)+q\right)^{\alpha}-\left(d\left(v_{i}\right)+3\right)^{\alpha}\right)-(q-3) 6^{\alpha} \\
\geq & (q-3)\left(4^{\alpha}-6^{\alpha}\right)+(q-1)\left((p+q)^{\alpha}-(p+3)^{\alpha}\right) \\
& +\left(\left(d\left(v_{q}\right)+q\right)^{\alpha}-\left(d\left(v_{q}\right)+3\right)^{\alpha}\right) \\
> & (q-3)\left(4^{\alpha}-6^{\alpha}\right)+(q-1)\left((p+q)^{\alpha}-(p+3)^{\alpha}\right) .
\end{aligned}
$$

Let $\phi(p, q)=(q-3)\left(4^{\alpha}-6^{\alpha}\right)+(q-1)\left((p+q)^{\alpha}-(p+3)^{\alpha}\right)$, where $p \geq 1, q \geq 4$ and $0<\alpha<1$. By Lemma 1 (the case of $k=q-3 \geq 1), \phi(p, q)$ is a strictly decreasing function on $p$. Since $0<\alpha<1$, then

$$
\frac{\partial \phi(p, q)}{\partial q}=\left((p+q)^{\alpha}-(p+3)^{\alpha}\right)+\alpha(q-1)(p+q)^{\alpha-1}+\left(4^{\alpha}-6^{\alpha}\right),
$$




$$
\begin{aligned}
\frac{\partial^{2} \phi(p, q)}{\partial q^{2}} & =\alpha(p+q)^{\alpha-2}((\alpha-1)(q-1)+2(p+q)) \\
& \geq \alpha(p+q)^{\alpha-2}(2 p+q+1)>0 .
\end{aligned}
$$

Thus, $\frac{\partial \phi(p, q)}{\partial q}$ is a strictly increasing function on $q$.

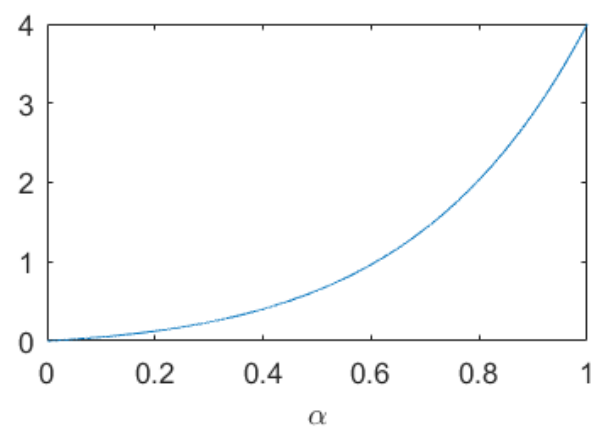

(a)

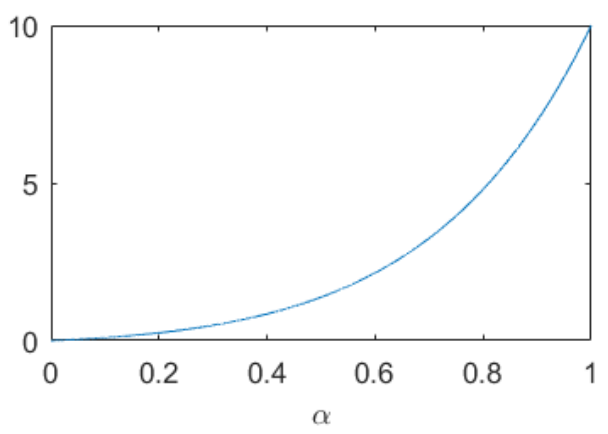

(c)

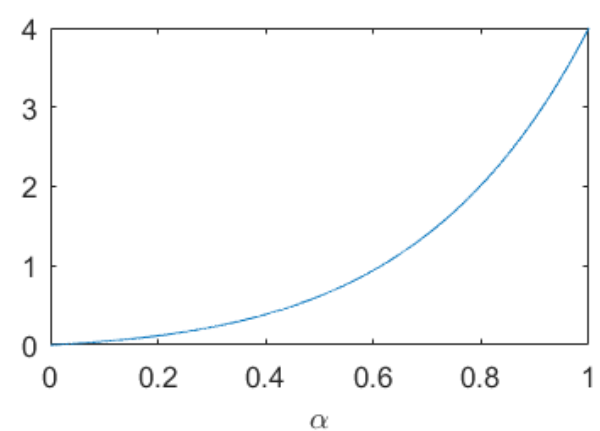

(b)

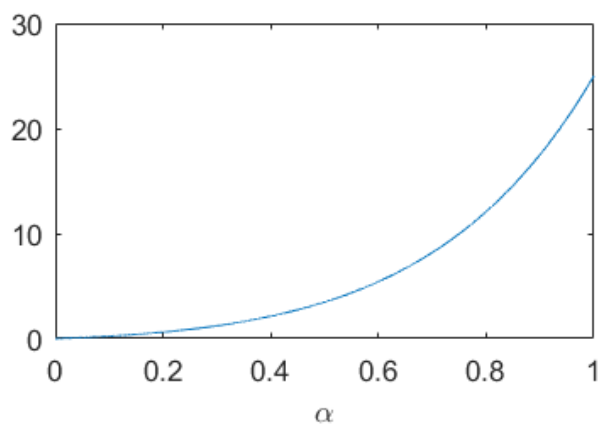

(d)

Figure 4. The four functions of $\alpha$ in Lemma 5.

We consider the following two cases:

Case 1. $T$ satisfies (i). In this case, we have $q \geq 5$ and $p \leq 3$, and so $\phi(p, q) \geq \phi(3, q)$. This implies that

$$
\begin{aligned}
\frac{\partial \phi(3, q)}{\partial q} & \geq\left.\frac{\partial \phi(3, q)}{\partial q}\right|_{q=5} \\
& =4 \alpha 8^{\alpha-1}+\left(4^{\alpha}-6^{\alpha}\right)+\left(8^{\alpha}-6^{\alpha}\right)=(8+4 \alpha) 8^{\alpha-1}+4^{\alpha}-2 \cdot 6^{\alpha}>0,
\end{aligned}
$$

where Figure 4 (a) implies the last strictly inequality. Therefore, $\phi(3, q)$ is a strictly increasing function on $q \geq 5$. Combining this with inequality (1), we obtain that

$$
\begin{aligned}
\chi_{\alpha}(T)-\chi_{\alpha}\left(T^{*}\right) & >\phi(p, q) \geq \phi(3, q) \geq \phi(3,5) \\
& =4\left(8^{\alpha}-6^{\alpha}\right)+2\left(4^{\alpha}-6^{\alpha}\right)=4 \cdot 8^{\alpha}+2 \cdot 4^{\alpha}-6^{\alpha+1}>0,
\end{aligned}
$$

where Figure 4 (b) implies the last strictly inequality.

Case 2. $T$ satisfies (ii). In this case, we have $q \geq 8$ and $p \leq 4$, and so $\phi(p, q) \geq \phi(4, q)$. This implies that

$$
\begin{aligned}
\frac{\partial \phi(4, q)}{\partial q} & \geq\left.\frac{\partial \phi(4, q)}{\partial q}\right|_{q=8} \\
& =7 \alpha 12^{\alpha-1}+\left(4^{\alpha}-6^{\alpha}\right)+\left(12^{\alpha}-7^{\alpha}\right) \\
& =(12+7 \alpha) 12^{\alpha-1}+4^{\alpha}-6^{\alpha}-7^{\alpha}>0,
\end{aligned}
$$


where Figure 4 (c) implies the last strictly inequality. Thus, $\phi(4, q)$ is a strictly increasing function on $q \geq 8$. By (1), we can conclude that

$$
\chi_{\alpha}(T)-\chi_{\alpha}\left(T^{*}\right)>\phi(p, q) \geq \phi(4, q) \geq \phi(4,8)=7\left(12^{\alpha}-7^{\alpha}\right)+5\left(4^{\alpha}-6^{\alpha}\right)>0,
$$

where Figure 4 (d) implies the last strictly inequality.

Let $P=u_{0} u_{1} \ldots u_{q}$ be a path of graph $G$. If $u_{0}$ is a pendant vertex of $G$ and $d\left(u_{1}\right)=d\left(u_{2}\right)=\cdots=$ $d\left(u_{q-1}\right)=2<3 \leq d\left(u_{q}\right)$ unless $q=1$, then we call $P$ as a pendant path of $G$. Hereafter, denote by $\mathcal{P}(G)$ the set of all pendant paths of $G$.

Lemma 6. [7] Let $T$ be a tree of $\mathcal{T}_{n, k}$. If $3 \leq k \leq\left\lfloor\frac{n+2}{3}\right\rfloor$ and $E_{2}(T) \subseteq E(\mathcal{P}(T))$, then

$$
\left|E_{2}(T)\right| \geq n_{4}+2 n_{5}+\cdots+(\Delta(T)-3) n_{\Delta(T)} .
$$

\section{Main results}

Now, we are ready to state the main result of this paper, that is,

Theorem 7. Let $T$ be a tree of $\mathcal{T}_{n, k}$. If $3 \leq k \leq\left\lfloor\frac{n+2}{3}\right\rfloor$ and $0<\alpha<1$, then

$$
\chi_{\alpha}(T) \geq k\left(3^{\alpha}+5^{\alpha}\right)+(n-3 k+2) 4^{\alpha}+(k-3) 6^{\alpha},
$$

with the equality iff $T \in \mathcal{T}_{n, k}^{*}$.

Proof. Throughout this proof, we suppose that $T^{*} \in \mathcal{T}_{n, k}$ has the smallest general sum-connectivity index. Since $k \geq 3$, then $\Delta\left(T^{*}\right) \geq 3$. Next, we prove some claims.

Claim 1. Every 2-vertex of $T^{*}$ is on a pendant path.

Proof of the Claim 1. By contradiction, assume that $u_{0}$ is not on any pendant path of $T^{*}$ with $d\left(u_{0}\right)=2$. In this case, each neighbor vertex of $u_{0}$ is a non-pendant vertex. In view of Lemma $2, \mathcal{T}_{n, k}$ contains a tree $T_{1}$ such that $\chi_{\alpha}\left(T^{*}\right) \geq \chi_{\alpha}\left(T_{1}\right)$, with equality iff $u_{0}$ is adjacent to at least one 2-vertex.

By applying the transformations in the proof of Lemma 2 repeatedly, a set of trees $\left\{T_{i} \mid i \geq 0\right\}$ belonging to $\mathcal{T}_{n, k}$ with $T_{0}:=T^{*}$ and a set of vertices $\left\{u_{i} \mid i \geq 0\right\}$ can be constructed such that each $u_{i}$ is a 2-vertex not on any pendant path of $T_{i}$ and $\chi_{\alpha}\left(T_{i}\right) \geq \chi_{\alpha}\left(T_{i+1}\right)$ for each $i \geq 0$.

Since the number of 2-vertices not on pendant paths of $T_{i+1}$ is one less than that of $T_{i}$, the above transformation will end after finite steps. That is, there is an integer $q \geq 0$ such that each 2-vertex of $T_{q+1}$ is on a pendant path. Therefore, $u_{q}$ is the unique 2-vertex of $T_{q}$ not on any pendant path, and hence $u_{q}$ is adjacent to two vertices with degrees at least 3 in $T_{q}$. Thus, we have $\chi_{\alpha}\left(T_{0}\right) \geq \chi_{\alpha}\left(T_{1}\right) \geq \cdots \geq \chi_{\alpha}\left(T_{q}\right)>\chi_{\alpha}\left(T_{q+1}\right)$, which is contrary with the choice of $T^{*}$.

By Claim 1, it follows that $E_{2}\left(T^{*}\right) \subseteq E\left(\mathcal{P}\left(T^{*}\right)\right)$. Next we shall prove that

$$
\Delta\left(T^{*}\right)=3 .
$$

By contradiction, we assume that (2) does not hold, that is, $\Delta\left(T^{*}\right) \geq 4$. From Lemma 6 , it follows that

$$
\left|E_{2}\left(T^{*}\right)\right| \geq n_{4}+2 n_{5}+\cdots+\left(\Delta\left(T^{*}\right)-3\right) n_{\Delta\left(T^{*}\right)} \geq \Delta\left(T^{*}\right)-3 \geq 1 .
$$

Suppose that $v_{0} \in T^{*}$ with $d\left(v_{0}\right)=\Delta\left(T^{*}\right) \geq 4$, and let $P:=v_{0} v_{1} \ldots v_{t}$ be a path of $T^{*}$ with $d\left(v_{t}\right) \geq 4$. We may assume that the length of $P$ is as large as possible. If $t=0$, then Lemmas 4 and $5(i)$ imply that $\mathcal{T}_{n, k}$ contains a tree with smaller general sum-connectivity index than $T^{*}$, but this contradicts the definition of $T^{*}$. Thus, $t \geq 1$. By Claim 1 , we have $\min \left\{d\left(v_{i}\right): 1 \leq i \leq t-1\right\} \geq 3$ when $t \geq 2$.

Let $N^{*}\left(v_{t-1}\right):=N\left(v_{t-1}\right)$ when $t=1$, and let $N^{*}\left(v_{t-1}\right):=N\left(v_{t-1}\right) \backslash\left\{v_{t-2}\right\}$ when $t \geq 2$. It is not difficult to see that $v_{t} \in N^{*}\left(v_{t-1}\right)$.

Claim 2. The degree of each vertex of $N^{*}\left(v_{t-1}\right)$ in $T^{*}$ is at most 4 and $d\left(v_{t}\right)=4$. 
Proof of Claim 2. Let $z \in N^{*}\left(v_{t-1}\right)$ and let $N(z)=\left\{z_{1}, z_{2}, \ldots, z_{q}\right\}$ with $d\left(z_{1}\right) \leq d\left(z_{2}\right) \leq \cdots \leq d\left(z_{q}\right)$. Clearly, $\left|E_{2}\left(T^{*}\right)\right| \geq \Delta\left(T^{*}\right)-3 \geq q-3$. Recall that the length of $P$ is as large as possible. Thus, $d\left(z_{q-1}\right) \leq 3$. Since $d\left(v_{t-1}\right)=\Delta\left(T^{*}\right) \geq 4>d\left(z_{q-1}\right)$ when $t=1$ (in this case, $\left.v_{t-1}=v_{0}\right)$ and $d\left(v_{t-1}\right) \geq 3 \geq d\left(z_{q-1}\right)$ when $t \geq 2$, we may suppose that $z_{q}=v_{t-1}$. By the choice of $T^{*}$ and in view of Lemma $5(i)$, we deduce that $d(z) \leq 4$. Note that $v_{t} \in N^{*}\left(v_{t-1}\right)$ and $d\left(v_{t}\right) \geq 4$, we have $d\left(v_{t}\right)=4$. This completes the proof of Claim 2 .

Now, we consider the vertex $v_{t-1}$. On the one hand, in view of Claim 2, all vertices of $N\left(v_{t-1}\right)$ except $v_{t-2}$ (when $t \geq 2$ ) have degrees at most 4 in $T^{*}$. Since Lemma 5 (ii) and in view of the choice of $T^{*}$, we can conclude that $d\left(v_{t-1}\right) \leq 7$. On the other hand, by the proof of Claim 2 we can deduce that the degree of each vertex of $N\left(v_{t}\right)$ except $v_{t-1}$ in $T^{*}$ is at most 3 . Now, in view of $d\left(v_{t}\right)=4$ and bearing in mind the choice of $T^{*}$, Lemma 4 implies that $d\left(v_{t-1}\right) \geq 6$. Consequently, we know that $6 \leq d\left(v_{t-1}\right) \leq 7$.
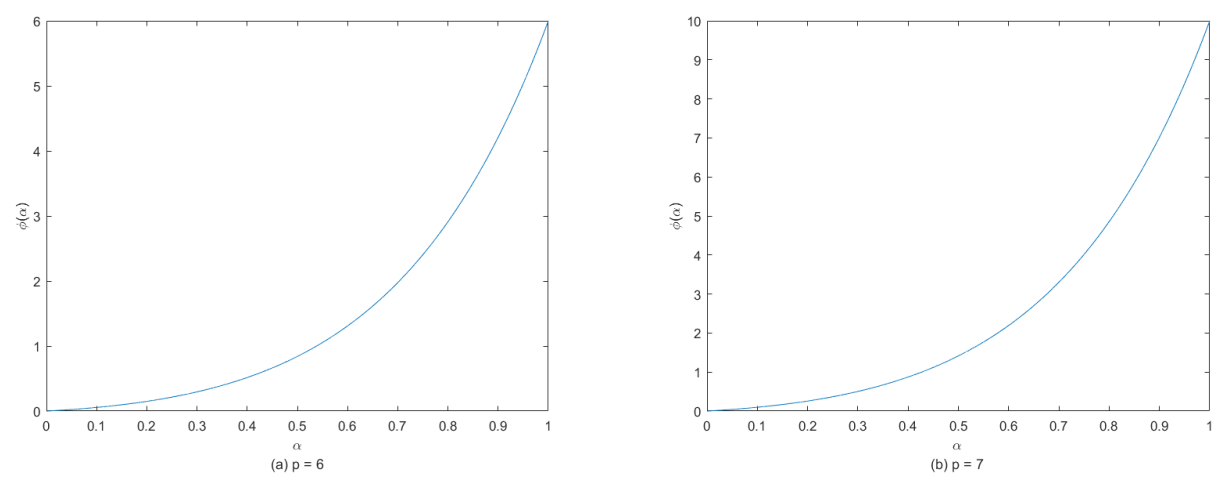

Figure 5. The function $\phi(\alpha)$ in Theorem 7.

Let $d\left(v_{t-1}\right)=p$ and let $N\left(v_{t-1}\right)=\left\{w_{1}, w_{2}, \ldots, w_{p}\right\}$ with $w_{p}:=v_{t-2}$ when $t \geq 2$. In view of Claim 2, it follows that $d\left(w_{i}\right) \leq 4$ for $1 \leq i \leq p-1$. Since $\left|E_{2}\left(T^{*}\right)\right| \geq 1$, we can construct a new tree $\bar{T}$ obtained after contracting an edge of $E_{2}\left(T^{*}\right)$ from $T^{*}$. By Transformation II, we can construct another tree $T^{\prime}:=\bar{T}_{v_{t-1} \hookrightarrow(3, p-3)}$ of $\mathcal{T}_{n, k}$ from $\bar{T}$. By Combining with $0<\alpha<1,6 \leq p \leq 7$ and Lemma 1 (the case of $k=p-4>1$ and $k=2$, respectively), we have

$$
\begin{aligned}
\chi_{\alpha}\left(T^{*}\right)-\chi_{\alpha}\left(T^{\prime}\right)= & 4^{\alpha}+\sum_{i=1}^{3}\left(\left(d\left(w_{i}\right)+p\right)^{\alpha}-\left(d\left(w_{i}\right)+4\right)^{\alpha}\right) \\
& +\sum_{i=4}^{p}\left(\left(d\left(w_{i}\right)+p\right)^{\alpha}-\left(d\left(w_{i}\right)+p-2\right)^{\alpha}\right)-(p+2)^{\alpha} \\
\geq & 4^{\alpha}+3\left((p+4)^{\alpha}-8^{\alpha}\right)+(p-4)\left((p+4)^{\alpha}-(p+2)^{\alpha}\right) \\
& +\left(\left(d\left(w_{p}\right)+p\right)^{\alpha}-\left(d\left(w_{p}\right)+p-2\right)^{\alpha}\right)-(p+2)^{\alpha} \\
> & 4^{\alpha}+3\left((p+4)^{\alpha}-8^{\alpha}\right)+(p-4)\left((p+4)^{\alpha}-(p+2)^{\alpha}\right)-(p+2)^{\alpha} \\
= & (p-1)(p+4)^{\alpha}+4^{\alpha}-3 \cdot 8^{\alpha}-(p-3)(p+2)^{\alpha} .
\end{aligned}
$$

Let $\phi(\alpha)=(p-1)(p+4)^{\alpha}+4^{\alpha}-3 \cdot 8^{\alpha}-(p-3)(p+2)^{\alpha}$, where $0<\alpha<1$. If $p=6$, then $\phi(\alpha)>0$, where Figure 5 (a) implies the inequality. If $p=7$, then $\phi(\alpha)>0$, where Figure 5 (b) implies the inequality. Now, we can conclude that $\chi_{\alpha}\left(T^{*}\right)-\chi_{\alpha}\left(T^{\prime}\right)>0$ whenever $6 \leq p \leq 7$, a contradiction. So, (2) holds.

By (2), we have $n_{1}+n_{2}+n_{3}=n$ and $n_{1}+2 n_{2}+3 n_{3}=2(n-1)$. Note that $n_{1}=k$ and $3 \leq k \leq\left\lfloor\frac{n+2}{3}\right\rfloor$. Thus, $n_{2}=n-2 k+2 \geq k$. Now, in view of Claim 1 and (2), to finish our proof, it suffices to show that each pendant vertex is adjacent to a 2-vertex. By contradiction, we assume that there is a pendant vertex $u$ of $T^{*}$ such that $u$ is adjacent to a 3 -vertex. Since $n_{2} \geq k$ and in view of Claim 1 , we have $E_{2}\left(T^{*}\right) \neq \varnothing$. By the choice of $T^{*}$ and in view of Lemma 3, it will deduce a contradiction. Thus, every pendant vertex of $T^{*}$ must be adjacent to a 2-vertex. By a simple calculation, we have

$$
\chi_{\alpha}\left(T^{*}\right)=k\left(3^{\alpha}+5^{\alpha}\right)+(n-3 k+2) 4^{\alpha}+(k-3) 6^{\alpha} .
$$


This cpmpletes the proof of the theorem.

In view of Theorem 7 and since $\mathcal{T}_{n, k}^{*} \subseteq \mathcal{C} \mathcal{T}_{n, k} \subseteq \mathcal{T}_{n, k}$, we can deduce the following result for chemical trees immediately.

Theorem 8. Let $T$ be a tree of $\mathcal{C} \mathcal{T}_{n, k}$. If $0<\alpha<1$ and $3 \leq k \leq\left\lfloor\frac{n+2}{3}\right\rfloor$, then

$$
\chi_{\alpha}(T) \geq k\left(3^{\alpha}+5^{\alpha}\right)+(n-3 k+2) 4^{\alpha}+(k-3) 6^{\alpha},
$$

with the equality iff $T \in \mathcal{T}_{n, k}^{*}$.

Acknowledgments: The author thanks sincerely the anonymous referee and Professor Muhuo Liu for their valuable comments, which lead to an improvement of the presentation of this paper.

Conflicts of Interest: "The author declare no conflict of interest."

\section{References}

[1] Gutman, I., \& Trinajstić, N. (1972). Graph theory and molecular orbitals. Total $\pi$-electron energy of alternant hydrocarbons. Chemical Physics Letters, 17(4), 535-538.

[2] Li, X., \& Zheng, J. (2005). A unified approach to the extremal trees for different indices. MATCH Commun. Math. Comput. Chem, 54(1), 195-208.

[3] Zhou, B., \& Trinajstić, N. (2010). On general sum-connectivity index. Journal of mathematical chemistry, 47(1), $210-218$.

[4] Cui, Q., \& Zhong, L. (2017). On the general sum-connectivity index of trees with given number of pendent vertices. Discrete Applied Mathematics, 222, 213-221.

[5] I. Tomescu, \& M. Arshad. (2015). On general sum-connectivity index of connected unicyclic graphs with $k$ pendant vertices. Discrete Applied Mathematics, 181 (30), 306-309.

[6] Tomescu, I., \& Kanwal, S. (2013). Ordering trees having small general sum-connectivity index. MATCH Commun. Math. Comput. Chem, 69(3), 535-548.

[7] Zhang, L. Z., Lu, M., \& Tian, F. (2007). Maximum Randić index on trees with $k$-pendant vertices. Journal of mathematical chemistry, 41(2), 161-171.

(C) 2019 by the authors; licensee PSRP, Lahore, Pakistan. This article is an open access article distributed under the terms and conditions of the Creative Commons Attribution (CC-BY) license (http://creativecommons.org/licenses/by/4.0/). 